\title{
Spatiotemporal Mode-Locking in a Fiber Laser
}

\author{
$\underline{\text { Vladimir L. Kalashnikov }}^{1, *}$ and Stefan Wabnitz ${ }^{1,2}$ \\ ${ }^{1}$ Dipartimento di Ingegneria dell'Informazione, Elettronica e Telecomunicazioni, Sapienza Universit a di \\ Roma, via Eudossiana 18, 00184 Rome, Italy \\ ${ }^{2}$ Novosibirsk State University, Pirogova 1, Novosibirsk 630090, Russia \\ *E-mail: vladimir.kalashnikov@uniroma1.it
}

DOI: 10.31868/RFL2020.98

The endeavor of multidimensional soliton generation in nonlinear optics (socalled "light bullets") and liquid crystals, Bose-Einstein condensates, etc., has a long history ${ }^{1-4}$. Such coherent and strongly localized structures could provide unprecedented energy (or mass) condensation, bridging across micro- and macro-scaled phenomena. The study of multidimensional solitons introduces a new branch of "mesoscopic" physics, permitting the study of a broad area of nonlinear phenomena far from thermodynamic equilibrium.

We introduce a spatiotemporal mode-locking mechanism in a fiber (or waveguide) laser, based on nonlinear mode-cleaning enhanced by graded dissipation. Our analysis is based on the generalized dissipative Gross-Pitaevskii equation, which has a broad impact on nonlinear physics, including nonlinear optics and Bose-Einstein condensates. We demonstrate that careful control of dissipative and non-dissipative physical mechanisms results in the self-emergence of stable $(2+1)$-dimensional dissipative solitons. Achieving such a regime does not require any additional mode-locking mechanisms, and allows for stable energy (or "mass") harvesting by coherent localized structures, such as ultrashort laser pulses or Bose-Einstein condensates.

We anticipate that the nonlinear coupling of spatial modes in either graded-index or photonic-crystal fibers, supported by the presence of graded dissipation, could implement the concept of the distributed Kerr-lens mode-locking 5 in a fiber laser in the regime of multimode self-cleaning. That would provide a means to achieve highlyefficient and stable energy harvesting in an all-fiber laser, without the need of using any additional mode-locking mechanisms. In a broader context, we envisage that photonic devices could provide an efficient tool for metaphorical or analog modeling of strongly localized coherent (or partially coherent) structures, which spontaneously emerge in nonlinear nonequilibrium dissipative systems. In particular, these systems represent a classical analog of a Bose-Einstein condensate in the weakly-dissipative limit.

\section{Acknowledgments}

This work has received funding from the European Union Horizon 2020 research and innovation program under the Marie Sklodowska-Curie grant No. 713694 (MULTIPLY), the ERC Advanced Grant No. 740355 (STEMS), and the Russian Ministry of Science and Education Grant No. 14.Y26.31.0017.

\section{References}

[1] B. A. Malomed, Eur. Phys. J. Special Topics 225, 2507-2532 (2016)

[2] Y. V. Kartashov et al, Nature Reviews 1,185-197 (2019)

[3] K. Krupa et al, APL Photonics 4,110901 (2019)

[4] V. N. Serkin, T. L. Belyaeva, Optik $176,38-48$ (2019)

[5] J. Zhang et al, Advanced Solid State Lasers Conference, OSA Technical Digest, ATh4A.7 (2015) 